\title{
Australia's national mental health strategy in historical perspective: beyond the frontier
}

\author{
Alan Rosen \\ Director of Clinical Services and Senior Psychiatrist, Royal North Shore Hospital and Community Mental Health \\ Services, Sydney, New South Wales, Australia, email arosen@nsccahs.health.nsw.gov.au
}

he history of Australian psychiatry is entwined with the impact of European (British) invasion and settlement, initially in 1788 , to form penal colonies to alleviate the overcrowding of English jails, which generated a masculine-dominated, individualistic culture. As European settlement in Australia expanded, the colonisers tried to come to terms with this remote, vast landscape and fought over land and resources with the original Aboriginal inhabitants, who had been there between 40000 and 60000 years. Australian psychiatry was profiled in a previous article in International Psychiatry (issue 10, October 2005).

\section{Deinstitutionalisation}

Australia's first large institution opened in 1838, with many more emerging over the next 100 years.

The development of psychiatric in-patient units at local general hospitals initially did not shift the concentration of work with in-patients with severe mental illness from the psychiatric hospitals, as the units were initially highly selective and would not take involuntary patients.

Australia also developed a substantial private medical sector, funded publicly through the Health Insurance Commission as well as through private health insurance schemes, although the sector has increasingly dealt with less severe disorders.

Meanwhile, from the early 1970s, some community health teams were put in place nationally, but they were often idealistically focused on primary prevention, and offered mainly generic services within office hours only.

\section{Recent national developments}

The National Mental Health Policy (1992) was endorsed by all Australian health ministers. It provided transitional funding in the national budget through the accompanying National Mental Health Strategy to shift services from institutions to local communities (see Fig. I). This strategy has enjoyed bipartisan political support, although the federal funding has been reduced in recent years. There have been three distinct phases of the National Mental Health Strategy (Department of Health and Aging, 2005).

First National Mental Health Plan (1993-98)

In the First National Mental Health Plan, services were to be shifted from stand-alone psychiatric hospitals to become largely community-based, 'mainstreamed' services, that is, integrated with and accessible via general health services, although remaining distinct as specialised mental health services. These included seven day and night per week mobile communitybased mental health crisis intervention services, assertive community treatment teams, a range of supervised community residential facilities, community vocational rehabilitation services and social recovery services, integrated with local psychiatric in-patient units based in general hospitals. They were to develop strong links with groups of consumers, families, general practitioners, the non-government service organisations, and other non-health services, such as housing, disability services, social security and employment. Initiatives included:

o promoting consumer and carer participation in policy and planning at every level, including direct ministerial access

O a Mental Health Category Classification and Costing Study (MHCASC) of whole episodes of psychiatric care, as an alternative or adjunct to hospital-based case mix (Buckingham et al, 1998)

o community awareness mass media campaign and studies of community and staff attitudes to people with mental illness (Rosen et al, 2000)
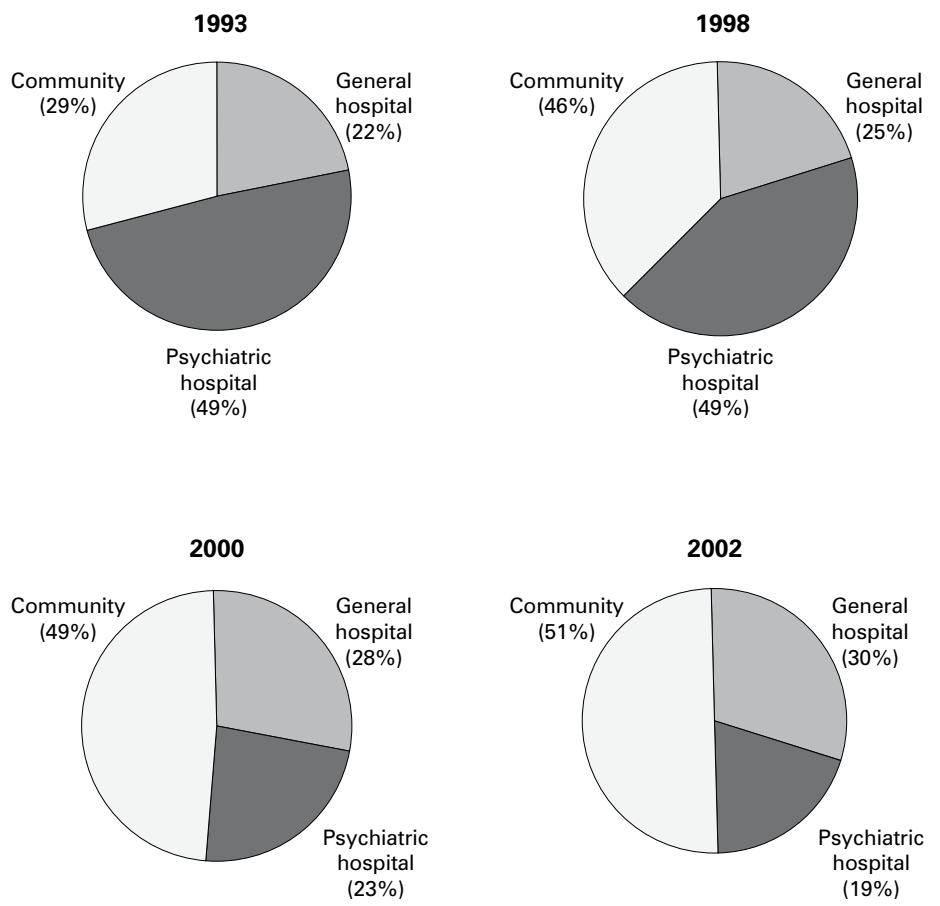

Fig. I Change in the composition of public mental health expenditure, 1993-2002.
Meanwhile, from

the early 1970s, some community

health teams

were put in place

nationally, but

they were often

idealistically

focused

on primary

prevention, and

offered mainly

generic services within office hours only. 
O developing national mental health standards (Gianfrancesco et al, 1996), which are now being used as the basis for accreditation surveys.

In relation to the last point, most Australian mental health services have now been surveyed for accreditation with the standards at least once. The adoption of the standards by the largest independent organisation for accreditation of healthcare services, public and private, has entailed the training and participation of paid consumer and family carer surveyors among the network of health professional surveyors.

\section{Second National Mental Health Plan (1998-2003)}

The Second National Mental Health Plan focused on the principles of mental health promotion, prevention, partnerships with other (non-health) providers of services, and quality. Initiatives included the following.

o Principles were developed for workforce planning, and more recently national workforce standards, defining the core practical skills all mental health professionals should have and use (Department of Health and Family Services, 2002).

There is now broad agreement that the Third Plan is far too superficial, provides few incentives to the states, even to ensure that they complete the first two Plans, and lacks real accountability mechanisms.

o Further anti-stigma strategies were put in place, such as the dissemination of media kits (now called Mindframe) to assist the press to put a more constructive 'spin' on suicide and mental illness stories, a 'Mind Matters' programme of mental health and illness education for all school students, and a manual for consumer advocates. Rotary International local branches now sponsor mental health community awareness workshops throughout Australia, and 'beyondblue' provides a national depression awareness campaign (http://www. beyondblue.org.au).

O Early prevention, improved detection, early intervention and shared mental healthcare were encouraged on the part of general practitioners, for patients in all age-groups and with all mental disorders, but particularly for depression and psychosis in young people, and others at risk of suicide. Dedicated 'early intervention in psychosis' teams have developed throughout Australia following the pioneering lead of McGorry et al (http://www.orygen.org.au).

- A National Mental Health and Well-being Community-wide Survey was undertaken for both high- and low-prevalence psychiatric disorders

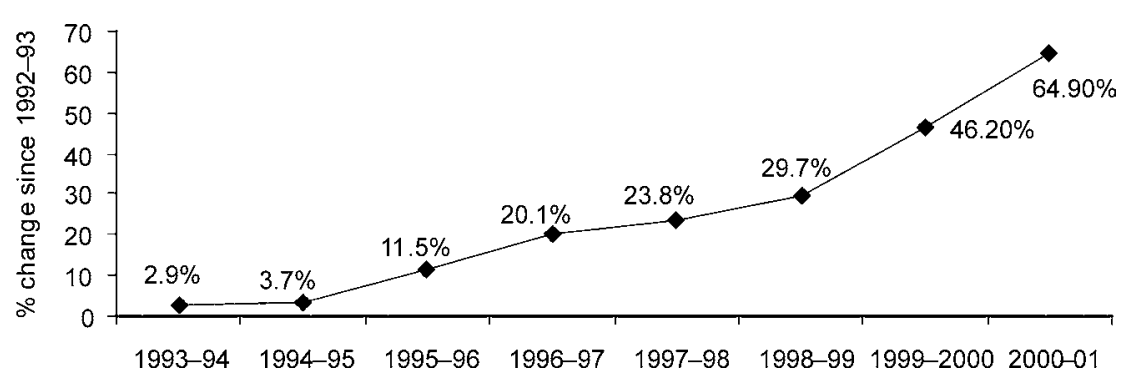

Fig. 2 Growth in total recurrent spending on specialised public mental health services (however, by comparison, the growth in general health spending over the same period was $66.1 \%$ ).
Box 1 Achievements of the First and Second Australian National Mental Health Plans

- Australian government recurrent spending on mental health services (2002-03), public and private, including pharmaceutical subsidies, was $A \$ 3183$ billion, or $A \$ 156$ per capita ( $A \$ 1$ is US\$0.7, €0.6).

- Average state spending on mental health services by 2003 was $A \$ 100.02$ per capita, a $32 \%$ increase (1993-2003) once adjusted for population growth and 2003 currency (see also Fig. 2).

- Public sector psychiatric beds decreased from $45 / 100000$ in 1993 to $31.4 / 100000$ in 2003, a $31 \%$ decrease overall, including a slight increase since 2001.

- Psychiatric beds in psychiatric hospitals as a percentage of total in-patient beds decreased from $76 \%$ in 1993 to $39 \%$ in 2003 , a $48.6 \%$ decrease overall, although they began to increase again after 2001-02.

- Consumer participation in decision-making, in terms of the percentage of mental health service organisations with formal participation mechanisms for specific mental health consumer representation, increased from $33 \%$ in 1993 to $68 \%$ in 2003.

- 'Level 1' consumer participation (i.e. employing someone in local services to represent the interests of mental health consumers and carers with service management) improved from $17 \%$ in 1993 to $53 \%$ in 2003 .

- Direct care staffing (in full-time equivalents) increased by 27\% from 1993 (14050) to 2003 (17950), with in-patient staffing decreasing by $10 \%$ and ambulatory and residential care staffing increasing by $120 \%$, to $87.1 / 100000$

Source: National Mental Health Report, 2005, from http://www health.gov.au/internet/wcms/publishing.nsf/Content/mentalnmhr05

(Whiteford \& Buckingham, 2005), which demonstrated that most psychiatric care is still inequitably distributed by general practitioners.

O A national suite of clinical, functional and self-report outcome measures was mandated for use in all public and private mental health services and facilities, with a national training and data management network (http://www.mhnocc.org).

The achievements of the First and Second National Mental Health Plans are summarised in Box I and Figs I and 2.

\section{Third National Mental Health Plan (2003-08)}

The Third National Mental Health Plan was recently adopted by all the Australian federal and state governments (Australian Health Ministers' Advisory Committee, 2003). It is to be guided by four priority themes: promoting mental health and preventing mental health problems and mental illness; increasing service responsiveness; strengthening quality; and fostering research, innovation and sustainability.

There is now broad agreement that the Third Plan is far too superficial, provides few incentives to the states, even to ensure that they complete the first two Plans, and lacks real accountability mechanisms. Accordingly, the substantial early achievements 
of the National Strategy are beginning to fray, and gaps in services are appearing as evidence-based community services are diluted and/or retracted increasingly to hospital sites. There has also been an unanticipated growth in acute presentations of comorbid drug misuse and mental illness (Gurr, 2005), leading to access block in emergency and inpatient departments.

Media glare on the human consequences of these problems has led recently to serial national inquiries by the Mental Health Council of Australia (Groom et al, 2003), the Human Rights and Equal Opportunity Commission (2005) and the Australian Senate (2006). This pressure prompted the prime minister to announce a substantial injection of funding, largely to privatised services for higher-prevalence mental health disorders, and to challenge the states politically to match it with enhancements for core public services for lower-prevalence severe conditions, which they are unlikely to attempt more than gesturally. Further attention is likely to be paid to early intervention in suicide prevention with young people.

\section{Forums for reform}

The Mental Health Services Conference of Australia and New Zealand (http://www.themhs.org) is a strong independent movement (Andrews, 2005) comprising all mental health professions, managers, consumers, family and indigenous networks. It organises binational forums for evidence dissemination and debate, to promote mental health service reform.

\section{Conclusions}

Firstly, we should acknowledge that there is common ground between all people of all cultural backgrounds in Australia - we have all had the experience of living on the margins at some stage, or in some generation of our family lives in Australia. This requires supporting the struggle for full citizenship and rights for people who are still on the margins of society, including Aboriginal people, detained asylum seekers and people living with mental illnesses (Rosen, 2006).

Secondly, it is clear from the Australian example that sustained national mental health reform is achievable; that structural reform of mental health services is easier to achieve than improvements in service quality; and that the support of clinicians, consumers and carers is a critical factor in the success of mental health reforms (Whiteford \& Buckingham, 2005).

Mental health reform in Australia looks good on paper, has been heading broadly in an appropriate direction and has achieved international recognition. However, these reforms are already losing momentum. Core local mental health services are being eroded or have never developed widely enough. After a period of sustained growth in spending on mental health services (Fig. 2), Australia now lags behind similar Western countries in terms of the proportion of gross domestic product and health budget spent on, and government funding of, mental health services (Rosen et al, 2004; Hickie et al, 2005; Rosen, 2006), and will still do so, even with recently announced enhancements.

There is further concern that the closing of institutions in Australia has been half-hearted and incomplete; that it has not been accompanied by full transfer of real investment in mental health services and facilities; and that under-resourced services are again being expected to be everything to everyone (Rosen et al, 2004; Hickie et al, 2005; Rosen, 2006).

Finally, there is a need for more coherent coordination of mental health services at one level of government (Andrews, 2005) and an independent National Mental Health Commission (Rosen et al, 2004; Hickie et al, 2005) similar to the potent standing commission operating in New Zealand, to monitor reforms externally, to cost the gaps in services, and to represent mental health service use needs directly to government.

\section{References}

Andrews, G. (2005) Editorial. The crisis in mental health: the chariot needs one horseman. Medical Journal of Australia, 182 372-373.

Australian Health Ministers' Advisory Committee (2003) National Mental Health Plan 2003-2008. Canberra: Commonwealth of Australia.

Australian Senate (2006) A National Approach to Mental Health - From Crisis to Community. http://www.aph.gov.au/senate/ committee/mentalhealth_ctte/report02/report.pdf

Buckingham, W., Burgess, P., Solomon, S., et al (1998) Developing a Case-Mix Classification for Mental Health Services. Vol. I: Main Report. Canberra: Commonwealth Department of Health and Family Services.

Department of Health and Aging (2005) National Mental Health Report 2005. Summary of Ten Years of Reform Under the National Mental Health Strategy 1993-2003. Canberra: Commonwealth of Australia.

Department of Health and Family Services (2002) National Practice Standards for the Mental Health Workforce. National Mental Health Strategy. Canberra: Commonwealth of Australia.

Gianfrancesco, P., Miller, V., Rauch, A., et al (1996) National Standards for Mental Health Services. Canberra: Australian Health Ministers National Mental Health Working Group.

Groom, G., Hickie, I. \& Davenport, T. (2003) 'Out of Hospital, Out of Mind!' A Report Detailing the Mental Health Services in Australia in 2002 and Community Priorities for National Mental Health Policy for 2003-2008. Canberra: Mental Health Council of Australia.

Gurr, R. (2005) Drugs and Australasian government policies have created a crisis in public mental health services. Submission to the Senate Select Committee on Mental Health Services from CASP: Comprehensive Area Service Psychiatrists' Network, 2005. http:// www.aph.gov.au/senate/committee/mentalhealth ctte

Hickie, I., Groom, G., McGorry, P., et al (2005) Australian mental health reform: time for real outcomes. Medical Journal of Australia, 182, 401-406.

Human Rights and Equal Opportunity Commission (2005) Not for Service: Experiences of Injustice and Despair in Mental Health Care in Australia. Canberra: Mental Health Council of Australia.

Rosen, A. (2006) The Australian experience of deinstitutionalization: the effect of Australian culture on the development and reform of its mental health services. Acta Psychiatrica Scandinavica, I I 3 (suppl. 429), I-9.

Rosen, A., Walter, G., Casey, D., et al (2000) Combating psychiatric stigma: an overview of contemporary initiatives. Australasian Psychiatry, 8, 19-26.

Rosen, A., McGorry, P., Groom, G., et al (2004) Australia needs a Mental Health Commission. Australasian Psychiatry, 12, 213-219.

Whiteford, H. \& Buckingham, W. J. (2005) Ten years of mental health service reform in Australia: are we getting it right? Medical Journal of Australia, I82, 396-400
Mental health

reform in Australia

looks good on

paper, has been

heading broadly

in an appropriate

direction and

has achieved

international

recognition.

However, these reforms are

already losing

momentum.

Acknowledgements: I am grateful to the Australian National Mental Health report 2005 for data in Box 1 and Figs 1 and 2; to Acta Psychiatrica Scandinavica for permission to use some material included in Rosen (2006), to Sylvia Hands for the typing and Karen Signorio and Kingsley Waterson fo organising Box 1 and Figs 1 and 2; and also to Professor Bruce Singh, Melbourne, and Viv Miller, Sydney, for assistance with the content. 